\title{
Alteração refracional após capsulotomia posterior com laser Nd:YAG
}

\author{
Change on refraction after $\mathrm{Nd}$ :YAG laser posterior capsulotomy

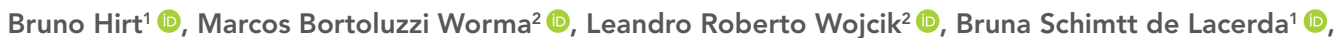 \\ Luciane Bugmann Moreira' ${ }^{1}$, Peter Alexander von Harbach Ferenczy ${ }^{1}$ [D

\section{Descritores:}

Capsulotomia posterior: Refração; Catarata;

Facoemulsificação

Keywords:

Posterior capsulotomy; Refraction; Cataract; Phacoemulsification

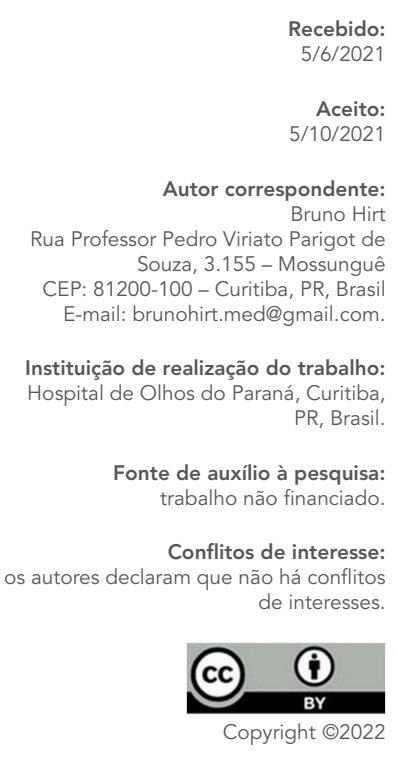

Recebido: pondente: Souza, 3.155 - Mossunguê P: 81200-100 - Curitiba, PR, Brasil trabalho não financiado.

os de interesse: há conflitos

\section{RESUMO}

Objetivo: Identificar se há mudança refracional significativa após realização de capsulotomia posterior com laser Nd:YAG em olhos pseudofácicos.

Métodos: Estudo retrospectivo com análise de prontuários de pacientes atendidos em um hospital com diagnóstico de opacificação de cápsula posterior do cristalino tratada com capsulotomia posterior com laser Nd:YAG no período de outubro de 2019 a março de 2021. A comparação entre a refração antes e após o procedimento foi realizada calculando-se o equivalente esférico. Também foi avaliada a mudança da acuidade visual, aferida por LogMAR.

Resultados: Foram analisados 90 prontuários, totalizando 140 olhos, de pacientes submetidos à capsulotomia posterior com laser $\mathrm{Nd}$ :YAG. O equivalente esférico médio pré-procedimento foi de $-0,07 \pm 0,89 \mathrm{D}$, mínimo de $-3,0 \mathrm{D}$ e máximo de $+2,5 \mathrm{D}$, mediana (intervalo interquartil) de $0,0 \mathrm{D}(-0,50 \mathrm{D}$ a +0,375D). A média pós-procedimento foi de -0,18 $00,86 \mathrm{D}$, mínimo de $-3,5 \mathrm{D}$ e máximo de $+2,25 \mathrm{D}$, mediana (intervalo interquartil) de $-0,125 \mathrm{D}(-0,50 \mathrm{D}$ a $0,0 \mathrm{D})$. com $\mathrm{p}<0,0082$. Dos 140 olhos, 66 sofreram miopização e 37 hipermetropização. A média de alteração do equivalente esférico geral foi de $-0,12 \pm 0,51 \mathrm{D}$, mínimo de $-2,50 \mathrm{D}$ e máximo de $+1,25 \mathrm{D}$, mediana (intervalo interquartil) de $0,0 \mathrm{D}(-0,375 \mathrm{D}$ a $+0,125 D$ ). Ao se comparar a diferença entre o equivalente esférico antes e após o procedimento do grupo de olhos que sofreu miopização $(n=66)$ ou hipermetropização $(n=37)$, separadamente, ambos obtiveram $p<0,0001$. Ao se compararem todos os olhos que sofreram alguma alteração refracional ( $n=103)$, foi encontrado $p=0,008$. A acuidade visual média pré-procedimento foi de $0,23 \pm 0,32$, mínimo de 0,0 e máximo de 2,3. Pós-procedimento, a média foi de 0,06 $\pm 0,13$, mínimo de $-0,12$ e máximo de 0,7 , com $p<0,0001$

Conclusão: A capsulotomia posterior com laser $\mathrm{Nd}$ :YAG gerou melhora significativa da acuidade visual nos pacientes do estudo, porém também gerou alteração refracional significativa após o procedimento, tanto para miopização (a mais frequente), quanto para hipermetropização.

\section{ABSTRACT}

Objective: To identify if there is a significant change on refraction after Nd:YAG laser posterior capsulotomy in pseudophakic eyes.

Methods: A retrospective study with analysis of medical records of patients treated at a hospital, with diagnosis of opacification of posterior lens capsule treated with $\mathrm{Nd}$ :YAG laser posterior capsulotomy, from October 2019 to March 2021. The comparison of refraction before and after the procedure was performed by calculating the spherical equivalent. Changes in visual acuity (VA), measured by LogMAR, were also evaluated.

Results: A total of 90 medical records (140 eyes) of patients submitted to $\mathrm{Nd}$ :YAG laser posterior capsulotomy were analysed. The mean pre-procedure spherical equivalent was $-0.07 \pm 0.89 \mathrm{D}$, minimum of $-3.0 \mathrm{D}$ and maximum of $+2.5 \mathrm{D}$, median (interquartile range) of $0.0 \mathrm{D}(-0.50 \mathrm{D}$ to $+0.375 \mathrm{D})$. The postprocedure mean was $-0.18 \pm 0.86 \mathrm{D}$, minimum of $-3.5 \mathrm{D}$ and maximum of $+2.25 \mathrm{D}$, median (interquartile range) of $-0.125 \mathrm{D}(-0.50 \mathrm{D}$ to $0.0 \mathrm{D})$, with $\mathrm{p}<0.0082$. Of the 140 eyes, 66 underwent myopia and 37 hyperopia, the mean change in the general spherical equivalent was $-0.12 \pm 0.51 \mathrm{D}$, minimum $-2.50 \mathrm{D}$ and maximum $+1.25 \mathrm{D}$, median (interquartile range) of $0.0 \mathrm{D}(-0.375 \mathrm{D}$ to $+0.125 \mathrm{D})$. When comparing the difference between the spherical equivalent before and after the procedure of the group of eyes that underwent myopia $(n=66)$ or hyperopia $(n=37)$, separately, both obtained $p<0.0001$. When comparing all eyes that suffered any change on refraction $(n=103)$, the $p$ value was 0.008 . The mean pre-procedure visual acuity was $0.23 \pm 0.32$, minimum of 0.0 and maximum of 2.3. After the procedure, the mean was $0.06 \pm 0.13$, minimum of -0.12 and maximum of $0.7, p<0.0001$

Conclusion: Nd:YAG laser posterior capsulotomy significantly improved visual acuity of patients in this study; however, it also led to a significant change on refraction after the procedure, both for myopization, which was more frequent, and for hyperopization. 


\section{INTRODUÇÃO}

A opacidade de cápsula posterior (OCP) é a complicação mais comum da cirurgia de catarata, com incidência relatada de $10 \%$ a 50\%. ${ }^{(1)}$ Uma metanálise publicada em $1998^{(2)}$ relatou incidência de OCP de $12 \%$ no primeiro ano, $21 \%$ no terceiro ano e de $28 \%$ no quinto ano após a cirurgia de catarata.

A causa da OCP é multifatorial, sendo descrita mais frequentemente como um crescimento de células epiteliais do cristalino que formam pérolas de fibrose e geram a opacidade. Remanescentes de células epiteliais cristalinianas também podem estar envolvidos na patogênese, assim como depósitos de proteínas ou células brancas do sangue provenientes de inflamação posterior. Distorções mecânicas no saco capsular ou roturas podem causar irregularidade, prejudicando a transparência na cápsula posterior. ${ }^{(1-3)}$ Ademais, alguns estudos comparam a incidência da OCP de acordo com a técnica cirúrgica e o material e desenho da lente intraocular (LIO). (4-6) $^{(2)}$

Redução na acuidade visual, perda de sensibilidade ao contraste, glare e diplopia monocular são os sintomas mais relacionados à OCP. O tratamento padrão-ouro é a capsulotomia posterior com laser Nd:YAG, sigla para neodímio dopado ítrio alumínio garnet ou, em inglês, neodymium-doped yttrium aluminum garnet. Trata-se de um laser de estado sólido, com taxa de sucesso de mais de $95 \% .{ }^{(7)}$ As principais complicações relacionadas ao procedimento são o dano da LIO, o aumento transitório da pressão intraocular (PIO), o edema macular cistoide, a alteração no vítreo anterior e o aumento da incidência de descolamento de retina. ${ }^{(3)}$

Contudo, a capsulotomia posterior também pode induzir um pequeno movimento, posterior ou anterior, da LIO, sendo que o tamanho da capsulotomia interfere na movimentação da lente, ou seja, capsulotomias maiores induzem mais deslocamento, e capsulotomias menores são recomendadas, para evitar tal complicação. (3,7,8) $^{(3)}$

Até o momento, há poucos estudos publicados sobre o efeito da capsulotomia posterior com laser Nd:YAG na alteração refracional causada por deslocamento da LIO. Não há evidência na literatura de alteração significativa da refração após o procedimento, porém existem alguns relatos de deslocamento da LIO para a câmara posterior após capsulotomia. ${ }^{(5,7,9,10)}$ Entretanto, vê-se a falta de embasamento na literatura, visto que são escassos os relatos de caso e estudos comparativos com LIOs e aparelhos Nd:YAG modernos. ${ }^{(3)}$

O objetivo deste estudo foi identificar se há mudança refracional após realização de capsulotomia posterior com laser Nd:YAG e verificar a significância estatística dos achados, comparando com os dados da literatura.

\section{MÉTODOS}

Este foi um estudo retrospectivo, aprovado pelo Comitê de Ética em Pesquisa do Hospital de Olhos do Paraná, em Curitiba (PR) com CAAE 46035021.8.0000.0093. A coleta e a avaliação de informações de saúde dos pacientes neste estudo aderiram aos princípios da Declaração de Helsinque.

Foram coletados dados dos prontuários de pacientes atendidos no Setor de Catarata do hospital, no período de outubro de 2019 a março de 2021. Foram incluídos pacientes que apresentaram pelo menos um dos olhos pseudofácicos, história e diagnóstico de OCP tratada com capsulotomia posterior com laser Nd:YAG, cuja LIO estivesse posicionada no saco capsular com cápsula posterior íntegra previamente ao procedimento e cujo procedimento de capsulotomia posterior com laser Nd:YAG tivesse ocorrido sem intercorrências.

Foram analisados dados como idade e sexo do paciente, tempo entre a cirurgia de catarata e a realização de capsulotomia posterior com laser Nd:YAG, tipo de LIO implantada, acuidade visual antes e após o procedimento medida por tabela de Snellen (valores posteriormente convertidos para LogMAR por meio do cálculo do $\log _{10}$ da divisão do denominador pelo numerador de Snellen) e refração antes e após o procedimento. Pacientes cujas cápsulas posteriores tivessem sido rompidas na cirurgia de catarata e cuja LIO estivesse localizada no sulco ciliar foram excluídos do estudo.

Todos os pacientes realizaram acuidade visual com a melhor correção antes do procedimento e 7 a 30 dias após o procedimento (autorrefração seguida por refração subjetiva). A comparação entre a refração pré e pós-procedimento se utilizou do cálculo do equivalente esférico, somando-se a refração esférica com a metade da refração cilíndrica.

Todos realizaram o procedimento com o mesmo cirurgião no aparelho Ellex Tango ${ }^{\mathrm{TM}}$ (Ellex, Adelaide, Austrália), sendo realizados no mínimo 20 disparos, de 2,2mJ cada, na cápsula posterior dos pacientes, utilizando-se a técnica em cruz. Fez-se uso de anestesia tópica ocular com cloridrato de oxibuprocaína $4 \mathrm{mg} / \mathrm{mL}$ e midríase medicamentosa com tropicamida 10mg $/ \mathrm{mL}$.

Os dados obtidos foram analisados estatisticamente com o programa computacional BioEstat v.5.3.o. (Belém, Brasil), utilizando o teste $t$ para amostras pareadas. Foi adotado o nível de significância de 5\%. 


\section{RESULTADOS}

Foram analisados 90 prontuários, totalizando 140 olhos, de pacientes submetidos à capsulotomia posterior com laser Nd:YAG. Do total de pacientes, 52 (58\%) eram mulheres, e a idade média foi de $67,8 \pm 11,6$ anos. A mediana (intervalo interquartil - IIO) do tempo decorrido, em meses, entre a cirurgia de catarata e a capsulotomia posterior com laser Nd:YAG foi de 15,8 (6,1 a 28,2) meses, com mínimo de 23 dias e máximo de 125,8 meses. Em relação ao lado operado, ocorreram 71 (50,7\%) procedimentos no lado direito (Tabela 1).

Tabela 1. Dados epidemiológicos

\begin{tabular}{lc} 
Dados & \\
Pacientes & 140 \\
Olhos & \\
Sexo & $38(42,2)$ \\
$\quad$ Masculino & $52(57,8)$ \\
$\quad$ Feminino & \\
Olho operado & $71(50,7)$ \\
Direito & $69(49,3)$ \\
Esquerdo & \\
Idade, meses & $67,8 \pm 11,6$ \\
$\quad$ Geral & $77,2 \pm 5,2$ \\
$\quad$ Homens & $60,8 \pm 9,9$ \\
Mulheres & $15,8(6,1-28,2)$ \\
\hline
\end{tabular}

Resultados expressos por $n, n(\%)$, média \pm desvio-padrão ou mediana (intervalo interquartil).

O equivalente esférico médio pré-procedimento foi de -0,07£0,89D, com mínimo de -3,00D e máximo de $+2,50 \mathrm{D}$, mediana (IIQ) de 0,00D (-0,50D a +0,375D). A média do equivalente esférico pós-procedimento foi de

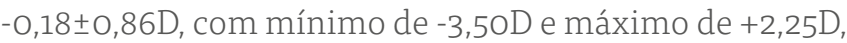
mediana (IIQ) de -0,125D (-o,50D a 0,0oD). A média da diferença geral dos equivalentes esféricos foi de -0,12 $\pm 0,51 D$, com mínimo de $-2,50 \mathrm{D}$ e máximo de $+1,25 \mathrm{D}$, mediana (IIQ) de o,ooD (-0,375D a +0,125D), com p<0,0082 (Tabela 2 e Figura 1).

Tabela 2. Refração pré e pós-procedimento, com n=140

\begin{tabular}{lccc} 
& Antes & Depois & $\begin{array}{c}\text { Valor } \\
\text { de } \mathbf{p}\end{array}$ \\
\hline $\begin{array}{l}\text { Equivalente Esférico pré e pós- } \\
\text { YAG (em dioptrias) }\end{array}$ & & \\
Média \pm DP & $-0,07 \pm 0,89$ & $-0,18 \pm 0,86$ & $0,0082^{*}$ \\
Mediana (IIQ) & $0,0(-0,50-+0,375)$ & $-0,125(-0,50-0,0)$ & \\
Mínimo-máximo & $-3,0-+2,5$ & $-3,50-+2,25$ & \\
$\begin{array}{l}\text { Diferença entre o EE geral pré e } \\
\text { pós YAG }\end{array}$ & & \\
Média \pm DP & $-0,12 \pm 0,51 D$ & & \\
Mediana (IIQ) & $0,00(-0,375-+0,125)$ & \\
\hline *Teste tpareado & &
\end{tabular}

Dos 140 olhos, 66 sofreram miopização e 37 hipermetropização. Analisando apenas o grupo de olhos que sofreu miopização ( $\mathrm{n}=66)$, observaram-se médias pré-YAG

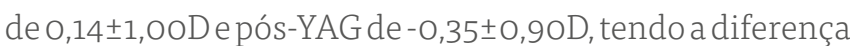

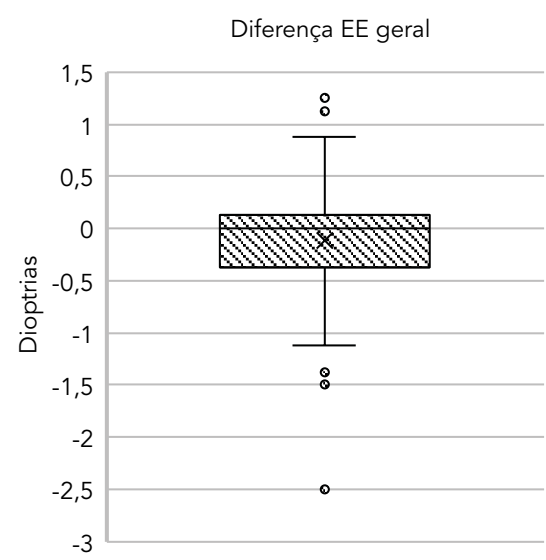

Box-plot indicando média, desvio-padrão, mediana, quartis e pontos de exceção.

E: equivalente esférico.

Figura 1. Distribuição dos valores relacionados à diferença de todos os equivalentes esféricos antes e após capsulotomia posterior com laser Nd:YAG.

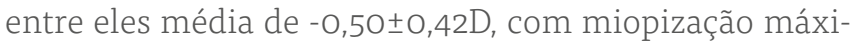
ma de $-2,50 D$ em um olho ( $\mathrm{p}<0,0001)$. Analisando apenas o grupo de olhos que sofreu hipermetropização ( $n=37$ ), observou-se média pré-YAG de -0,33£0,72D e pós-YAG de $0,13 \pm 0,79$, tendo a diferença entre eles média de

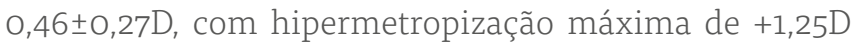
em um olho ( $\mathrm{p}<0,0001)$ (Figura 2). Ao se compararem todos os olhos que sofreram alguma alteração refracio-

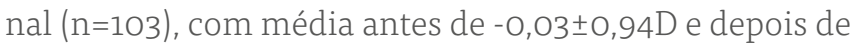
$-0,18 \pm 0,91 \mathrm{D}$, foi encontrado $\mathrm{p}=0,008$ (Tabela 3).

Tabela 3. Alteração refracional após YAG laser

\begin{tabular}{|c|c|c|}
\hline \multicolumn{3}{|l|}{ Alteração refracional pós-YAG (em dioptrias) } \\
\hline \multicolumn{3}{|l|}{ Miopização em 66 olhos } \\
\hline Máxima (n) & \multicolumn{2}{|c|}{$-2,50(1)$} \\
\hline Média \pm DP antes & $0,14 \pm 1,0$ & \multirow{3}{*}{$\mathrm{p}<0,0001^{*}$} \\
\hline Média \pm DP depois & $-0,35 \pm 0,9$ & \\
\hline Média \pm DP da diferença antes/depois & $-0,50 \pm 0,42$ & \\
\hline \multicolumn{3}{|l|}{ Hipermetropização em 37 olhos } \\
\hline Máxima (n) & \multicolumn{2}{|c|}{$+1,25(1)$} \\
\hline Média \pm DP antes & $-0,33 \pm 0,72$ & \multirow{3}{*}{$p<0,0001$} \\
\hline Média \pm DP depois & $0,13 \pm 0,79$ & \\
\hline Média \pm DP da diferença antes/depois & $0,46 \pm 0,27$ & \\
\hline \multicolumn{3}{|l|}{ Qualquer alteração refracional em 103 olhos } \\
\hline Média \pm DP antes & $-0,03 \pm 0,94$ & \multirow{2}{*}{$p=0,008^{\star}$} \\
\hline Média \pm DP depois & $-0,18 \pm 0,91$ & \\
\hline Sem alteração refracional & \multicolumn{2}{|c|}{37 olhos } \\
\hline
\end{tabular}

Em relação à acuidade visual, medida por LogMAR, a média pré-procedimento foi de 0,23 $\pm 0,32$, com mínimo de o,0 e máximo de 2,3. No pós-procedimento, a média foi de 0,06₫0,13, com mínimo de -0,12 e máximo de 0,7. A média da diferença entre a acuidade visual antes e após o YAG foi de -0,16 $\pm 0,31$ pontos LogMAR, com mediana (IIQ) de -0,08 (-0,18 a 0,0), mínimo de -0,4 e máximo de 0,12 

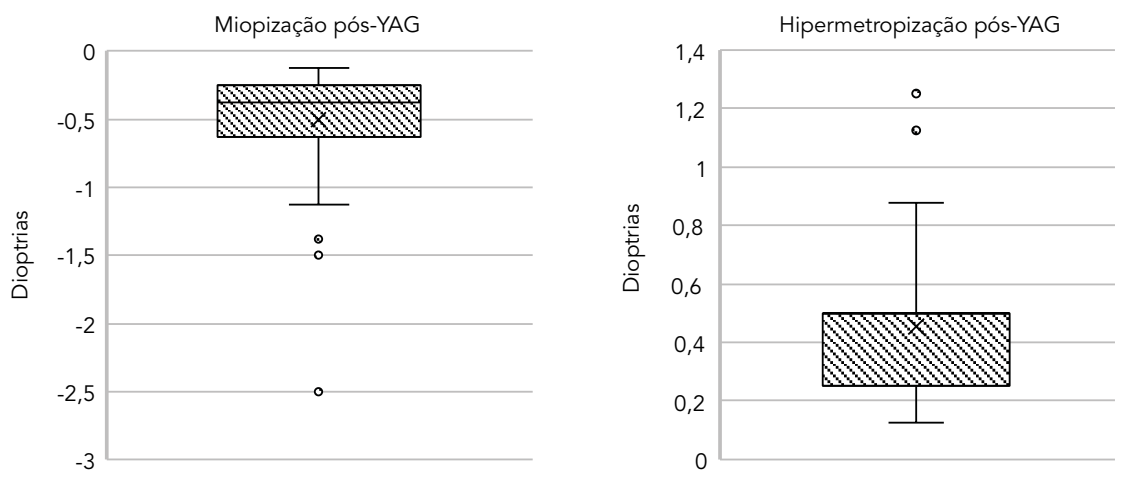

Box-plot indicando média, desvio-padrão, mediana, quartis e pontos de exceção.

Figura 2. Distribuição dos valores relacionados aos olhos que sofreram miopização e hipermetropização, respectivamente, após capsulotomia posterior com laser Nd:YAG.

(p<0,0001) (Tabela 4 e Figura 3). Dos 140 olhos, seis apresentaram piora da acuidade visual e, destes, dois apresentaram piora de 0,08 , três de 0,10 e um de 0,12.

Tabela 4. Acuidade visual pré e pós-procedimento, $n=140$

\begin{tabular}{lcccc}
\hline $\begin{array}{l}\text { Acuidade visual pré e pós- } \\
\text { YAG (LogMAR) }\end{array}$ & Antes & Depois & Diferença & $\begin{array}{c}\text { Valor } \\
\text { de } \mathbf{p}\end{array}$ \\
\hline Média \pm DP & $0,23 \pm 0,32$ & $0,06 \pm 0,13$ & $-0,16 \pm 0,31$ & \\
Mediana (IIQ) & $0,1(0,0-0,3)$ & $0,0(0,0-0,1)$ & $-0,08(-0,18-0,0)$ & $<0,0001^{*}$ \\
Mínimo e máximo & $0,0 \pm 2,3$ & $-0,12 \pm 0,7$ & $-0,4 \pm 0,12$ & \\
\hline
\end{tabular}

*Teste t pareado.

DP: desvio padrão; ॥Q: intervalo interquartil.

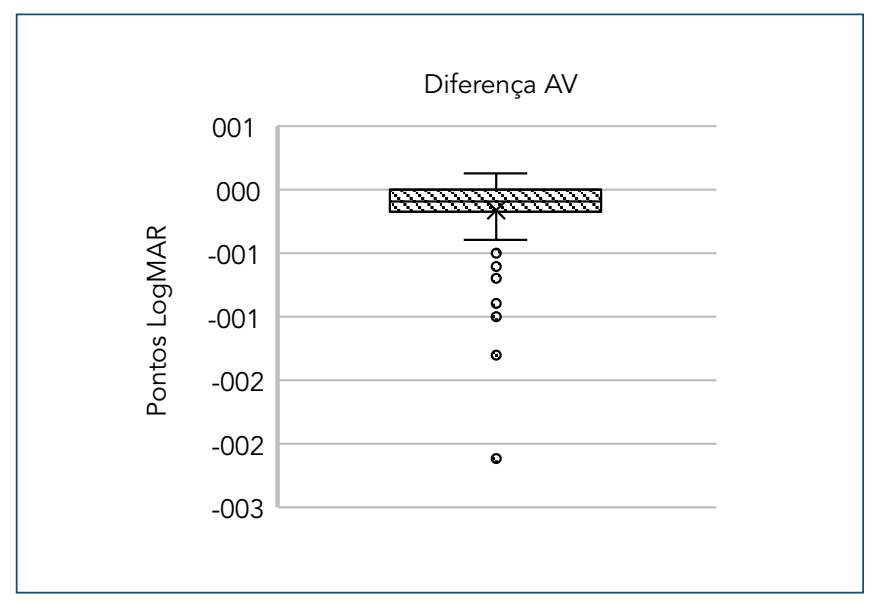

Box-plot indicando média, desvio-padrão, mediana, quartis e pontos de exceção.

AV: acuidade visual.

Figura 3. Distribuição dos valores relacionados à diferença da acuidade visual LogMAR entre pré e pós-capsulotomia posterior com laser $\mathrm{Nd}$ :YAG. Valores negativos indicam meIhora da acuidade visual.

\section{DISCUSSÃO}

Foi realizada uma análise de pacientes pseudofácicos, atendidos no Setor de Catarata do Hospital de Olhos do Paraná, que apresentavam opacificação de cápsula posterior, submetidos à capsulotomia posterior com Nd:YAG laser, a fim de avaliar a possibilidade de alteração refracional após o procedimento.
A capsulotomia posterior com laser Nd:YAG pode causar outras alterações, além da mudança refracional. ${ }^{(1-6)}$ Uma destas alterações é a elevação da PIO desde o procedimento até 4 horas após, retornando a valores pré-procedimento geralmente em 24 horas. Outra alteração importante a ser citada é o descolamento de retina, cujos fatores de risco incluem alta miopia, degeneração lattice com rotura associada, uso de alta energia no laser e capsulotomias grandes. Edema de córnea também pode ocorrer, o qual é descrito quando o laser acidentalmente atinge o plano da córnea ou quando o aumento da PIO pós-YAG gera edema. Casos de uveíte e vitreíte já foram descritos, podendo persistir até 6 meses após o procedimento, porém com taxas de incidência menores que 1\%. Danos à LIO também foram observados, desde quebras a marcações na LIO ocasionadas por aplicação do laser em um plano mais anterior, tendo taxa de incidência entre $4 \%$ a $40 \%$, a depender do material e do desenho da lente.

A mudança refracional após capsulotomia posterior com laser Nd:YAG já foi citada na literatura e pode ocorrer devido a um deslocamento no sentido anteroposterior da LIO após aplicação do laser. Alguns estudos observaram um deslocamento da LIO para posterior, hipermetropizando o paciente. ${ }^{(8-10)}$ Dados da literatura mostraram um possível deslocamento anterior da LIO, miopizando o paciente, porém, estatisticamente significativo em apenas um estudo, ${ }^{(5)}$ que o associou ao desenho da LIO.

Em relação ao equivalente esférico, os dados antes e após o YAG, quando analisados separadamente, têm pouca relevância, visto que são características próprias da amostra estudada. Contudo, quando se analisa a diferença entre eles, observou-se significância estatística para quaisquer alterações refracionais ( $\mathrm{p}<0,0001)$, corroborando outro estudo. ${ }^{(12)}$ Entretanto, demais trabalhos da literatura não encontraram significância nesse 
quesito. ${ }^{(5,7,7,9,11-13)}$ Os autores identificaram que, dos 140 olhos, 66 sofreram miopização, 37 hipermetropização e 37 não apresentaram alterações. Logo, prevaleceu a miopização da amostra.

Analisando apenas o grupo de olhos que sofreu miopização ( $\mathrm{n}=66 ; 47,1 \%)$, observou-se tendência de miopização de, em média, -0,50D ( $\mathrm{p}<0,0001)$, ao passo que o grupo que sofreu hipermetropização ( $\mathrm{n}=37 ; 26,4 \%)$ teve, em média, um acréscimo de +0,46D ( $\mathrm{p}<0,0001)$. Ao se analisarem todos os olhos que sofreram alguma alteração refracional (n=103; 73,6\%), observou-se média de mudança de -0,12D. Desse modo, pode-se inferir que houve significância estatística para alteração refracional dos olhos analisados $(\mathrm{p}=0,008)$, tanto para miopização quanto hipermetropização, com maior prevalência do primeiro. Apenas um estudo mostrou tal resultado. ${ }^{(12)}$ Demais trabalhos não encontraram significância estatística nesse quesito. ${ }^{(5,7,9,11-13)}$

Em relação à acuidade visual, foi observada melhora da acuidade visual em 79 (56,4\%) olhos, manutenção da acuidade visual prévia em 55 (39,3\%) olhos e piora da acuidade visual em seis (4,3\%) olhos. A média de diferença entre a acuidade visual antes e após o YAG foi de -0,16 pontos LogMAR. Logo, houve melhora significativa ( $\mathrm{p}<0,0001) \mathrm{da}$ acuidade visual na amostra analisada, assim como informado em outros estudos da literatura. ${ }^{(3,5,7,7,9,11-15)}$

Seis olhos apresentaram piora da acuidade visual. Destes, dois apresentaram piora de 0,08, três de 0,10 e um de o,12 ponto LogMAR, equivalentes, em cada caso, a piora de uma linha de visão. Uma revisão mostrou 3,5\% a 6\% de casos de piora da AV após laser Nd:YAG. ${ }^{(16)}$

Em relação ao tempo entre a cirurgia de catarata e a capsulotomia posterior com laser Nd:YAG, foi observada média de 27,2 meses, com a mais precoce ocorrendo apenas 23 dias após a cirurgia e a mais distante a aproximadamente 10,5 anos após. Não foram observadas relações estatisticamente significativas com idade e sexo. Achados da literatura mostram intervalos similarmente distribuídos entre a cirurgia de catarata e capsulotomia posterior com laser Nd:YAG. ${ }^{(5,7,7,11-15)}$

Em relação às LIOs, em 78 olhos foi possível identificar a LIO em uso, sendo elas: Rayner, RayOne ${ }^{\oplus}$, PanOptix LensX, PanOptix LensX Tórica, Eyeol LW, Eyeol Ultima, Mediphacos IOFlex, Acrysof ${ }^{\oplus}$ IQ, AcrySof ${ }^{\oplus}$ IQ Tórica, Abbott Tecnis ${ }^{\circledR}$ ZXR, Abbott Tecnis ${ }^{\circledR}$ ZLB, Abbott Tecnis ${ }^{\circledR}$ ZKB, Abbott Tecnis ZMB, ReSTOR e ReSTOR Tórica. Contudo, nos 62 olhos restantes, não foi possível identificar qual a LIO em uso, visto que esses pacientes não realizaram a cirurgia de catarata no mesmo serviço e, assim, não apresentam tais dados em prontuário. Vê-se, então, a possibilidade da realização de um estudo futuro abordando apenas os pacientes com dados da LIO em prontuário, buscando relações entre o desenho da lente e seu material com a mudança refracional, a acuidade visual e o tempo de aparecimento do OCP, assim como fez outro estudo da literatura. ${ }^{(5)}$

Existem algumas limitações neste artigo, como a incerteza relacionada ao modelo de LIO utilizada em alguns pacientes e, em alguns casos, da realização da refração pré-YAG diante presença de OCP, além do desenho retrospectivo do estudo, com coleta de dados baseada na análise de prontuários. Contudo, os resultados basearam-se em amostra de tamanho razoável para o tema, obtendo-se resultados mais fidedignos.

\section{CONCLUSÃO}

Após procedimento de capsulotomia com laser Nd:YAG, observou-se melhora significativa da acuidade visual, porém, também houve alteração refracional significativa após o procedimento, tanto para hipermetropização quanto para miopização, a qual se mostrou mais frequente na amostra estudada.

\section{REFERÊNCIAS}

1. Nguyen NX, Seitz B, Reese S, Langenbucher A, Küchle M. Accommodation after Nd: YAG capsulotomy in patients with accommodative posterior chamber lens 1CU. Graefes Arch Clin Exp Ophthalmol. 2005;243(2):120-6.

2. Schaumberg DA, Dana MR, Christen WG, Glynn RJ. A systematic overview of the incidence of posterior capsule opacification. Ophthalmology. 1998;105(7):1213-21.

3. Aslam TM, Devlin H, Dhillon B. Use of Nd:YAG laser capsulotomy. Surv Ophthalmol. 2003;48(6):594-612.

4. Kaindlstorfer $C$, Kneifl M, Reinelt P, Schönherr U. Rotation of a toric intraocular lens from neodymium:YAG laser posterior capsulotomy. J Cataract Refract Surg. 2018;44(4):510-1.

5. Monteiro T, Soares A, Leite RD, Franqueira N, Faria-Correia F, Vaz F. Comparative study of induced changes in effective lens position and refraction after $\mathrm{Nd}$ :YAG laser capsulotomy according to intraocular lens design. Clin Ophthal. 2018;12:533-7.

6. Thom H, Ender F, Samavedam S, Vivez CP, Gupta S, Dhariwal M, et al Effect of AcrySof versus other intraocular lens properties on the risk of $\mathrm{Nd}$ :YAG capsulotomy after cataract surgery: A systematic literature review and network meta-analysis. PLoS One. 2019;14(8):e0220498.

7. Parajuli A, Joshi P, Subedi P, Pradhan C. Effect of Nd:YAG laser posterior capsulotomy on intraocular pressure, refraction, anterior chamber depth, and macular thickness. Clin Ophthalmol. 2019;13:945-52.

8. Levy JH, Pisacano AM, Anello RD. Displacement of bag-placed hydrogel lenses into the vitreous following neodymium:YAG laser capsulotomy. J Cataract Refract Surg. 1990;16(5):563-6.

9. Thornval P, Naeser K. Refraction and anterior chamber depth before and after neodymium:YAG laser treatment for posterior capsule opacification in pseudophakic eyes: A prospective study. J Cataract Refract Surg. 1995;21(4):457-60.

10. Findl O, Drexler W, Menapace R, Georgopoulos M, Rainer G, Hitzenberger CK, et al. Changes in intraocular lens position after neodymium:YAG capsulotomy. J Cataract Refract Surg. 1999;25(5):659-62. 
11. Hu CY, Woung LC, Wang MC, Jian JH. Influence of laser posterior capsulotomy on anterior chamber depth, refraction, and intraocular pressure. J Cataract Refract Surg. 2000;26(8):1183-9.

12. Karahan E, Tuncer I, Zengin MO. The effect of ND:YAG laser posterior capsulotomy size on refraction, intraocular pressure, and macular thickness. J Ophthalmol. 2014;2014:846385.

13. Ozkurt YB, Sengör T, Evciman T, Haboğlu M. Refraction, intraocular pressure and anterior chamber depth changes after Nd:YAG laser treatment for posterior capsular opacification in pseudophakic eyes. Clin Exp Optom. 2009;92(5):412-5.
14. Keates RH, Steinert RF, Puliafito CA, Maxwell SK. Long-term followup of Nd:YAG laser posterior capsulotomy. J Am Intraocul Implant Soc. 1984;10(2):164-8.

15. Ari S, Cingü AK, Sahin A, Çinar Y, Çaça I. The effects of Nd:YAG laser posterior capsulotomy on macular thickness, intraocular pressure, and visual acuity. Ophthal Surg Las Im. 2012;43(5):395-400.

16. Weiblinger RP, Spring S. Review of the clnical literature on the use of the Nd:YAG laser for posterior capsulotomy. J Cataract Refract Surg. 1986;12(2):162-70. 